\title{
EFFICIENT COUNTERMEASURES AGAINST POWER ANALYSIS FOR ELLIPTIC CURVE CRYPTOSYSTEMS
}

Kouichi Itoh, Tetsuya Izu, and Masahiko Takenaka

FUJITSU LABORATORIES Ltd.

\{kito,izu,takenaka\}@labs.fujitsu.com

\begin{abstract}
The power analysis on smart cards is a real threat for cryptographic applications. In spite of continuous efforts of previous countermeasures, recent improved and sophisticated attacks against Elliptic Curve Cryptosystems are not protected. This paper proposes two new countermeasures, the Randomized Linearly-transformed Coordinates (RLC) and the Randomized Initial Point (RIP) against the attacks including the Refined Power Analysis (RPC) by Goubin and the Zero-value Point Analysis (ZPA) by Akishita-Takagi. Proposed countermeasures achieve notable speed-up without reducing the security level.
\end{abstract}

Keywords: Smart cards, power analysis, Elliptic Curve Cryptosystems, countermeasure

\section{Introduction}

Smart cards are becoming a new infrastructure in the coming IT society for their applications such as the SIM cards for mobile phones, identification cards for entrance systems and electronic tickets for movies. However, the power analysis attacks against these devices are real threats for these applications. In these attacks, an adversary observes traces of the power consumption of the device, and then, he detects a correlation between this information and some secret information hidden in the device. The simple power analysis (SPA) and the differential power analysis (DPA) are classical but typical examples [17, $18,22]$. Fortunately, various countermeasures which is not only secure but also efficient, have been proposed before 2002 [5, 15].

Recently, improved and sophisticated power analysis on Elliptic Curve Cryptosystems (ECC) have been proposed. In 2003, Goubin presented a new analysis, the Refined Power Analysis (RPA), which detects special points with 0coordinate on the curve by chosen messages [9]. Then, Akishita-Takagi extended RPA to the Zero-value Point Analysis (ZPA), which detects 0 value in additions and doublings [2]. Some previous countermeasures resist RPA 
and ZPA [1, 5, 6], however, they require larger amount of processing time. A practical countermeasure was proposed by Ciet-Joye [7]. Note that Smart's countermeasure resists only RPA [32]; it does not always resist ZPA [3].

In this paper, we propose two practical countermeasures for ECC, the Randomized Linearly-transformed Coordinates countermeasure (RLC) and the Randomized Initial Point countermeasure (RIP), which resist power analysis including above newer attacks, and provide efficient processing speed for scalar multiplications. Proposed countermeasures achieve notable speed-up without reducing the security level.

The rest of this paper is organized as follows: we briefly review Elliptic Curve Cryptosystems (ECC) in section 2. Side channel attacks and countermeasures are in section 3. Then section 4 describes our proposed countermeasures. A comparison of countermeasures are described in section 5. Concrete algorithms of proposed countermeasures are in the appendix.

\section{Elliptic Curve}

This section describes a brief review of elliptic curves defined over finite fields with prime elements. This is just for a simlicity and basic ideas of most attacks and countermeasures can be applied to other finite fields.

\section{Elliptic Curve Cryptosytems (ECC)}

Let $K$ be a finite field with prime elements $p>3$. An elliptic curve over $K$ is represented by an equation $E(K):=\left\{(x, y) \in K \times K \mid y^{2}=x^{3}+\right.$ $\left.a x+b, a, b \in K, 4 a^{3}+27 b^{2} \neq 0\right\} \cup\{\mathcal{O}\}$. The special point $\mathcal{O}$ is called the point at infinity. In this representation, a point on the curve (different from $\mathcal{O}$ ) is represented as a pair of $K$-elements (affine coordinates). An elliptic curve $E(K)$ forms an additive group by the following addition law: the point $\mathcal{O}$ is a neutral element, and an inverse point of $P=(x, y)$ is given by $-P=$ $(x,-y)$. We call $P_{1}+P_{2}\left(P_{1} \neq P_{2}\right)$ the elliptic curve addition (ECADD) and $P_{1}+P_{2}\left(P_{1}=P_{2}\right)$, that is $2 P_{1}$, the elliptic curve doubling (ECDBL). Let $P_{1}=\left(x_{1}, y_{1}\right), P_{2}=\left(x_{2}, y_{2}\right)$ be two elements of $E(K)$ different from $\mathcal{O}$ and satisfying $P_{1} \neq-P_{2}$. Then the addition $P_{3}=P_{1}+P_{2}=\left(x_{3}, y_{3}\right)$ is defined by $x_{3}=\lambda^{2}-x_{1}-x_{2}, \quad y_{3}=\lambda\left(x_{1}-x_{3}\right)-y_{1}$, where $\lambda=\left(y_{2}-y_{1}\right) /\left(x_{2}-x_{1}\right)$ for $P_{1} \neq P_{2}$, and $\lambda=\left(3 x_{1}^{2}+a\right) /\left(2 y_{1}\right)$ for $P_{1}=P_{2}$.

Elliptic Curve Cryptosystems (ECC) are one of the standard technologies in the area of cryptography [24]. The biggest advantage of ECC is the key length; it is currently chosen much shorter than that of existing other cryptosystems such as RSA and ElGamal. This feature is quite suitable for smart card implementations. Let $\boldsymbol{d}$ be an integer (scalar) and $P$ be a point on the elliptic curve $E(K)$. The point $P$ is called the base point. A scalar multiplication is an operation to compute a point $d P=P+\cdots+P(d-1$ additions). Computing $d$ 
Table 1. Add-and-double method

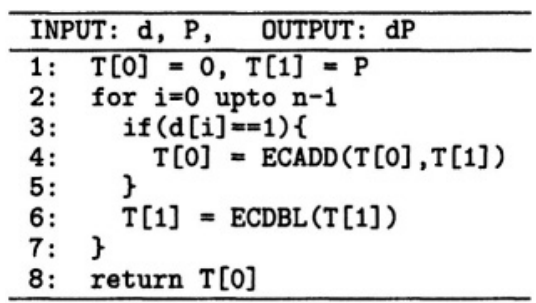

Table 2. Add-and-double-always method

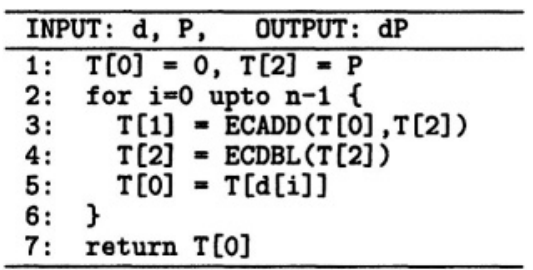

from $d P$ and $P$ is called the elliptic curve discrete logarithm problem, and the hardness of this problem assures the security of ECC. In most ECC schemes, $\boldsymbol{d}$ is used as a secret key. A dominant computation of all encryption/decryption and signature generation/verification algorithms of ECC is the scalar multiplication. Efficiency of a scalar multiplication strongly depends on a choice of addition chains and coordinate systems for the elliptic curve representation.

\section{Addition Chain and Coordinate System}

Let $d=d_{n-1} 2^{n-1}+\cdots+d_{1} 2^{1}+d_{0}$ be a binary expression of a scalar $d$ with $d_{n-1}=1$. Then the add-and-double (AD) method from the least significant bit (LSB) for a scalar multiplication is shown in Table 1, which requires $n-$ 1 ECDBLs and $n / 2$ ECADDs in average. The number of ECADDs can be reduced by using the signed-binary expression of $\boldsymbol{d}$, however, the technique is vulnerable to side channel attacks and we are not interested in it in this paper. A similar algorithm can be constructed from the most significant bit (MSB), however, we omit it because of the space limitation.

Coordinate systems for the elliptic curve representation effect on the processing speed of ECADD/ECDBL and hence a scalar multiplication [8]. In the affine coordinates $\mathcal{A}$, the addition formula always requires an inverse in the definition field $K$. In some environments, especially in prime fields (our situation), computing inverses are expensive. Since the projective coordinates and the Jacobian coordinates provide division-free formulas, these coordinates are widely used in practice. In the projective coordinates $\mathcal{P}$, a point is represented as a tuple $(X, Y, Z)$ where two points $(X, Y, Z)$ and $(\lambda X, \lambda Y, \lambda Z)\left(\lambda \in K^{*}\right)$ are identical. A curve equation is obtained by setting $x=X / Z, y=Y / Z$ in the curve equation. In the Jacobian coordinates $\mathcal{J}$, a point is also represented as a tuple $(X, Y, Z)$ where two points $(X, Y, Z)$ and $\left(\lambda^{2} X, \lambda^{3} Y, \lambda Z\right)(\lambda \in$ $\left.K^{*}\right)$ are identical. A curve equation is obtained by setting $x=X / Z^{2}, y=$ $Y / Z^{3}$ in the curve equation. The addition formulas for general cases (namely, $P_{1}, P_{2} \neq \mathcal{O}$ and $P_{1} \neq-P_{2}$ for ECADD) in the Jacobian coordinates are given in Table 3. Note that in these coordinates, the point at infinity is represented as a tuple, which is the only point whose $Z$-coordinate value equals 0 . 
Table 3. Addition formulas in the Jacobian coordinates (general cases)

\begin{tabular}{l} 
ECADD (in the Jacobian coordinates) \\
\hline Input: $P_{1}=\left(X_{1}, Y_{1}, Z_{1}\right), P_{2}=\left(X_{2}, Y_{2}, Z_{2}\right)$ \\
Output: $P_{3}=P_{1}+P_{2}=\left(X_{3}, Y_{3}, Z_{3}\right)$ \\
\hline$U_{1} \leftarrow X_{1} Z_{2}^{2}, U_{2} \leftarrow X_{2} Z_{1}^{2}, S_{1} \leftarrow Y_{1} Z_{2}^{3}$ \\
$S_{2} \leftarrow Y_{2} Z_{1}^{3}, W \leftarrow U_{2}-U_{1}, R \leftarrow S_{2}-S_{1}$ \\
$T \leftarrow U_{2}+U_{1}, M \leftarrow S_{2}+S_{1}$ \\
$X_{3} \leftarrow R^{2}-T W^{2}, V \leftarrow 3 T W^{2}-2 R^{2}$ \\
$Y_{3} \leftarrow\left(V R-M W^{3}\right) / 2, Z_{3} \leftarrow Z_{1} Z_{2}$ \\
\hline
\end{tabular}
ECDBL (in the Jacobian coordinates)

\begin{tabular}{l}
\hline Input: $P_{1}=\left(X_{1}, Y_{1}, Z_{1}\right), a$ \\
Output: $P_{4}=2 P_{1}=\left(X_{4}, Y_{4}, Z_{4}\right)$ \\
\hline$W \leftarrow a Z_{1}^{4}, M \leftarrow 3 X_{1}^{2}+W$ \\
$S \leftarrow 4 X_{1} Y_{1}^{2}, T \leftarrow 8 Y_{1}^{4}$ \\
$X_{4} \leftarrow M^{2}-2 S$ \\
$Y_{4} \leftarrow M\left(S-X_{4}\right)-T$ \\
$Z_{4} \leftarrow 2 Y_{1} Z_{1}$ \\
\hline
\end{tabular}

Table 4. Processing times of ECADD and ECDBL

\begin{tabular}{|c|c|c|c|c|}
\hline Coordinates & \multicolumn{2}{|c|}{ ECADD } & \multicolumn{2}{c|}{ ECDBL } \\
\cline { 2 - 5 } & $Z \neq 1$ & $Z=1$ & $a \neq-3$ & $a=-3$ \\
\hline $\mathcal{A}$ & $2 M+1 S+1 I$ & - & \multicolumn{2}{|c|}{$2 M+2 S+1 I$} \\
\hline $\mathcal{P}$ & $12 M+2 S$ & $9 M+2 S$ & $7 M+5 S$ & $7 M+3 S$ \\
\hline $\mathcal{J}$ & $12 M+4 S$ & $8 M+3 S$ & $4 M+6 S$ & $4 M+4 S$ \\
\hline
\end{tabular}

Required processing time of ECADD/ECDBL is summarized in Table 4, where $M, S, I$ denotes the processing time of a multiplication, a squaring, and an inverse in the definition field $K$, respectively. These processing time can be improved when one of $Z$-coordinate values in $\mathcal{P}, \mathcal{J}$ equals to 1 or the coefficient of the definition equation satisfies $a=-3$.

\section{Power Analysis}

The power analysis is a powerful attack against cryptographic schemes on some devices such as smart cards. An adversary observes leaked power consumption, and detects a correlation between this information and some secret information hidden in the device. The simple power analysis (SPA) [17] and the differential power analysis (DPA) [18,22] are typical examples.

\section{Simple Power Analysis}

The binary methods compute ECADD only when $d_{i}=1$. Hence an adversary easily detects this irregular procedure by observing power consumption and obtains the value of $d_{i}$. The attack is called the simple power analysis (SPA) [17]. A standard approach to resist SPA is to use so called the add-anddouble-always method (Table 2) [5], in which both ECADD and ECDBL are computed repeatedly (in step 3 and step 4). Since a pattern of the power trace becomes fixed, the adversary cannot detect the bit information $d_{i}$ by SPA. As a variant of this countermeasure, the Montgomery ladder (Table 5) [21] is sometimes used $[4,11,12,16,26,27]$, because it provides good processing speed by using the specialized addition formula, the $\boldsymbol{x}$-coordinate-only addition formula $[4,12,11]$ which will be discussed later. 
Table 5. Montgomery ladder

\begin{tabular}{|c|c|}
\hline \multicolumn{2}{|c|}{ INPUT: $d, P, \quad$ OUTPUT: $d P$} \\
\hline 1: & $T[0]=P$ \\
\hline 2: & $\mathrm{T}[1]=\operatorname{ECDBL}(\mathrm{T}[0])$ \\
\hline 3: & for $i=n-2$ downto 0\{ \\
\hline 4: & $\mathrm{T}[2]=\operatorname{ECDBL}(\mathrm{T}[\mathrm{d}[\mathrm{i}]])$ \\
\hline 5: & $=\operatorname{ECADD}(\mathrm{T}[0], \mathrm{T}[1])$ \\
\hline 6: & $=\mathrm{T}[2-\mathrm{d}[\mathrm{i}]]$ \\
\hline 7: & $=T[1+d[i]]$ \\
\hline 8: & \} \\
\hline 9: & return $\mathrm{T}[0]$ \\
\hline
\end{tabular}

Table 6. ADA and RPC

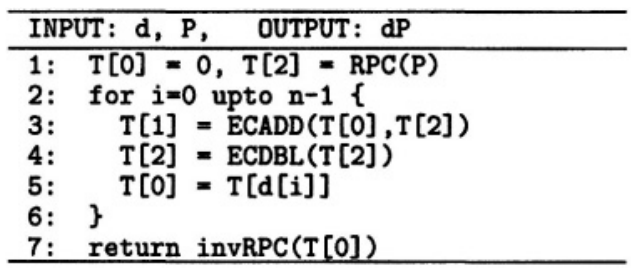

Note that some SPA-countermeasures use window-based methods for efficiency $[14,19,20,30,31]$. However, the memory requirement is severe in most cases on smart cards and we do not follow these approaches in this paper.

\section{Differential Power Analysis}

In the differential power analysis (DPA) [18, 22], an adversary guesses $d_{i}=$ 0 , for example, and simulates the computation repeatedly. Then he/she divides the power consumption into two sets depending on the assumption, in order to make a bias of hamming weights of the internal information between these sets. If the assumption is correct, a difference of the power consumption of two sets can be observed (as a spike) in the trace. Note that even if a scheme is SPA-resistant, it is not always DPA-resistant.

In order to resist DPA, a randomization of parameters is a well-known technique to make the simulation impossible [5]. One approach is to randomize a scalar $\boldsymbol{d}$ or an addition chain for $\boldsymbol{d}$. Coron proposed the scalar blinding countermeasure, in which $d$ is replaced by $d+r \phi$ for a random integer $r$ and the order of the point $\phi$ [5]. Original $r$ (20-bit) does not provide sufficient security [27]. Larger $r$ will provide higher security and less speed. Coron also proposed the base point blinding countermeasure, in which $d P$ is computed by $d P=d(P+R)-d R$ for a random point $R$. However biases are pointed out [27]. Oswald-Aigner used the randomized addition-subtraction chain [25], but effect of the randomization is not enough [28, 29, 33].

Clavier-Joye splits the scalar randomly, in which $d P$ is computed by $d P=$ $r P+(d-r) P$ for a random integer $r$ (the exponent splitting, ES) [6]. As no security problems have been pointed out, ES requires at least twice of the processing time than without it. Ciet-Joye proposed another splitting method, in which $d P$ is computed by $\lfloor d / r\rfloor(r P)+(d \bmod r) P$ for a random integer $r$ [7]. Here $r$ is as long as half of $\boldsymbol{d}$ and, with Shamir's trick, it provides practical processing speed without reducing the security.

Another approach for resisting DPA is to randomize a point representation. Coron proposed the randomized projective coordinates (RPC) countermeasure 
[5]. Let $P=(X, Y, Z)$ be a base point in the projective coordinates. Then $(X, Y, Z)$ equals to $(r X, r Y, r Z)$ for all $r \in K^{*}$ mathematically; but they all are different data as bit sequences. The power consumption of a scalar multiplication is randomized if $(X, Y, Z)$ is randomized to $(r X, r Y, r Z)$ for a randomly chosen integer $r \in K^{*}$. An example of RPC for the add-and-doublealways method is shown in Table 6 , where a function RPC outputs a randomized point by RPC and a function invRPC denotes its inverse function. Joye-Tymen proposed another DPA-countermeasure based on the randomization of point representation, the randomized curve (RC) countermeasure [15]. RC uses an isomorphism of elliptic curves with which a curve equation and a base point are transformed with holding the group structure. Again, let $P=(X, Y, Z)$ be a base point in the projective coordinates. Then $(X, Y, Z)$ and $\left(r^{2} X, r^{3} Y, Z\right)$ correspond to each other under the isomorphism. Two points are the same (under the isomorphism) mathematically; but different as bit sequences. Thus the power consumption will be randomized if the curve and the base point is randomized by a randomly chosen integer $r \in K^{*}$. A sample algorithm is easily obtained similarly to Table 6 by changing functions RPC, invRPC to $\mathrm{RC}$, invRC. RPC and RC provide efficient scalar multiplications because the conversions are quite cheap compared to the main multiplications.

Note that all countermeasures of ES, RPC, RC does not resist SPA. These countermeasures should be used combined with SPA-countermeasures.

\section{Refined Power Analysis and Zero-value Point Attack}

In 2003, Goubin presented a new power analysis against ECC, the refined power analysis (RPA), which reveals the secret scalar by detecting special points with 0-coordinates on the curve by chosen messages [9]. Let $E(K)$ be an elliptic curve which has the special point $Q_{y}=(0, y)$, for example. Suppose scalar multiplications are computed by add-and-double-always method from LSB (Table 2). When an adversary knows the bit information $d_{0}, \ldots, d_{j-1}$ of the secret key $d$, he/she tries to reveal the next bit $d_{j}$. At the end of the $(j-1)$ th loop in Table 2 , we have $T[2]=2^{j} P, T[0]=\sum_{i=0}^{j-1} d_{i} 2^{i} P$. Then, in the $j$-th loop, we will compute

$$
T[1] \leftarrow\left(\sum_{i=0}^{j-1} d_{i} 2^{i}+2^{j}\right) P, \quad T[0] \leftarrow \begin{cases}T[0]=\sum_{i=0}^{j-1} d_{i} 2^{i} P & \text { if } d_{j}=0 \\ T[1]=\left(\sum_{i=0}^{j-1} d_{i} 2^{i}+2^{j}\right) P & \text { if } d_{j}=1 .\end{cases}
$$

Then the adversary pre-computes $\bar{P}=\left(\sum_{i=0}^{j-1} d_{i} 2^{i}+2^{j}\right)^{-1} Q_{y}$, where the inverse is computed modulo the order of the curve, and computes a scalar multiplication on input $d$ and $\bar{P}$. If $d_{i}=1$, at the end of the $j$-th loop, we have $T[0]=\left(\sum_{i=0}^{j-1} d_{i} 2^{i}+2^{j}\right) \bar{P}=Q_{y}=(0, y)$ and the adversary easily detects $d_{i}$, because the power consumption of " 0 " is quite distinctive. This is a basic 
idea of RPA. The attack can be applied to the add-and-double-always method from MSB and the Montgomery ladder [9]. Note that the pre-computation is very easy for the adversary because elliptic curve parameters are public.

In the same year, Akishita-Takagi extended Goubin's RPA to the zero-value point attack (ZPA) [2]. A basic idea of ZPA is to observe 0 in the functions ECADD and ECDBL rather than in the addition chain where RPA paid attention. Akishita-Takagi discussed conditions when such 0 can be observed. In order to resist ZPA, implementers should avoid operations which possibly output 0 in ECADD and ECDBL.

\section{Countermeasures against RPA/ZPA}

A key of RPA is to observe 0 in the process. The most noteworthy property of RPA is that RPC and RC cannot resist RPA, because in RPC and RC, the special point $Q_{x}=(x, 0), Q_{y}=(0, y)$ are converted to point $(X, 0, Z),(0, Y, Z)$ by the conversion with keeping the specialty " 0 ". Because of the same reasons as RPA, RPC and RC cannot resist ZPA.

Smart proposed an RPA-countermeasures using isogeny of the curve [32]. However, the countermeasure does not resist ZPA [3]. Avanzi analyzed the resistance of Coron's scalar blinding and base point blinding countermeasure against RPA, and concluded that they are resistant to RPA [1]. Similarly, they also resist ZPA. However, these Countermeasures require larger amount of computing time than without them. The exponent splitting countermeasure [6] and its improved version [7] also resist both RPA and ZPA. Especially, CietJoye's countermeasure has almost no penalty from a viewpoint of efficiency.

\section{4. $\quad$ Proposed Countermeasures}

This section proposes two practical Countermeasures for ECC against power analysis including DPA, RPA and ZPA: the randomized linearly-transformed coordinates (RLC) and the randomized initial point (RIP) Countermeasures.

\section{Randomized Linearly-transformed Coordinates (RLC)}

Before proposing the countermeasure, we introduce the linearly-transformed coordinates (LC) in the following:

DEFINITION 1 In the linearly-transformed coordinates $(L C)$ with a parameterset $\left(\lambda_{X}(\lambda), \lambda_{Y}(\lambda), \lambda_{Z}(\lambda), \mu_{X}, \mu_{Y}, \mu_{Z}\right)$, apoint $(X, Y, Z)$ and $\left(X^{\prime}, Y^{\prime}, Z^{\prime}\right)$ are identified if there exists $\lambda \in K^{*}$ such that

$$
\begin{aligned}
X-\mu_{X} & =\lambda_{X}(\lambda)\left(X^{\prime}-\mu_{X}\right), \quad Y-\mu_{Y}=\lambda_{Y}(\lambda)\left(Y^{\prime}-\mu_{Y}\right) \\
Z-\mu_{Z} & =\lambda_{Z}(\lambda)\left(Z^{\prime}-\mu_{Z}\right),
\end{aligned}
$$

where $\lambda_{X}(\lambda), \lambda_{Y}(\lambda), \lambda_{Z}(\lambda)$ are functions of $\lambda$ and $\mu_{X}, \mu_{Y}, \mu_{Z} \in K$. 
Table 7. Addition formulas in LC for a parameter set $S_{1}=\left(\lambda^{2}, \lambda^{3}, \lambda, \mu, 0,0\right)$ (general case)

\begin{tabular}{l} 
LCECADD for $S_{1}=\left(\lambda^{2}, \lambda^{3}, \lambda, \mu, 0,0\right)$ \\
\hline Input: $P_{1}=\left(X_{1}, Y_{1}, Z_{1}\right)$, \\
\\
$P_{2}=\left(X_{2}, Y_{2}, Z_{2}\right), \mu$ \\
Output: $P_{3}=P_{1}+P_{2}=\left(X_{3}, Y_{3}, Z_{3}\right)$ \\
\hline$U_{1} \leftarrow\left(X_{1}-\mu\right) Z_{2}^{2}, U_{2} \leftarrow\left(X_{2}-\mu\right) Z_{1}^{2}$ \\
$S_{1} \leftarrow Y_{1} Z_{2}^{3}, S_{2} \leftarrow Y_{2} Z_{1}^{3}$ \\
$W \leftarrow U_{2}-U_{1}, R \leftarrow S_{2}-S_{1}$ \\
$T \leftarrow U_{2}+U_{1}, M \leftarrow S_{2}+S_{1}$ \\
$X_{3} \leftarrow R^{2}-T W^{2}+\mu$ \\
$V \leftarrow 3 T W^{2}-2 R^{2}$ \\
$Y_{3} \leftarrow\left(V R-M W^{3}\right) / 2, Z_{3} \leftarrow Z_{1} Z_{2} W$ \\
\hline
\end{tabular}

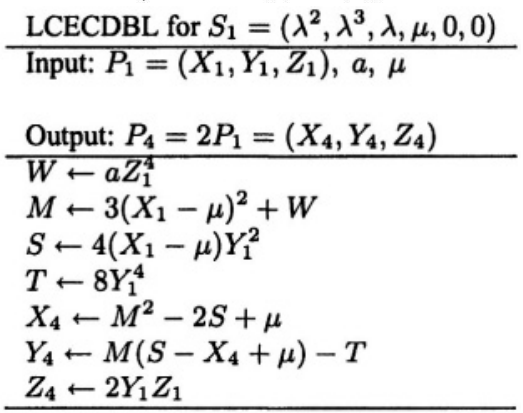

The projective coordinates $\mathcal{P}$ and the Jacobian coordinates $\mathcal{J}$ are examples of the linearly-transformed coordinates; the parameter set for $\mathcal{P}$ is $(\lambda, \lambda, \lambda, 0,0,0)$ and for $\mathcal{J}$ is $\left(\lambda^{2}, \lambda^{3}, \lambda, 0,0,0\right)$. One of the advantage of LC is a variation of representations. Especially, if we set $\mu_{X}, \mu_{Y} \neq 0$, the special point $Q_{y}=$ $(0, y)$ and $Q_{x}=(x, 0)$ are converted to some points whose coordinate values are different from 0 . However, the processing speed of ECADD/ECDBL become slower in general than previous coordinates.

As an example, we give explicit addition formulas LCECADD, LCECDBL which compute ECADD and ECDBL in the linearly-transformed coordinates for a parameter set $S_{1}=\left(\lambda^{2}, \lambda^{3}, \lambda, \mu, 0,0\right)$ in Table 7 . Here the coordinates are very similar to the formulas for the Jacobian case (Table 3 ).

Proposed Countermeasure. An idea of our countermeasure is quite simple. For given functions $\lambda_{X}(\lambda), \lambda_{Y}(\lambda), \lambda_{Z}(\lambda)$, we randomly choose $\mu_{X}, \mu_{Y}, \mu_{Z}$ and convert the base point into the transformed coordinates with the parameter set $\left(\lambda_{X}(\lambda), \lambda_{Y}(\lambda), \lambda_{Z}(\lambda), \mu_{X}, \mu_{Y}, \mu_{Z}\right)$ in the beginning of a scalar multiplication. After processing the scalar multiplication in LC, we re-convert the result to the original coordinates (randomized linearly-transformed coordinates, RLC). A generic algorithm of RLC is obtained by changing RPC, invRPC to RLC, invRLC, where a function RLC converts a point $P$ into a point with a chosen parameter set, and invRLC re-converts to the affine coordinates. Note that RLC can be applied to all addition chains mentioned in section 2.

Let us consider the security of RLC. Basically, we have to use the add-anddouble-always methods or the Montgomery ladder for the addition chain, for RLC does not resist SPA itself. On the other hand, RLC resists DPA if a parameter set is properly chosen, because RLC is a natural extension of RPC. But RPA will be successful when we choose $\mu_{X}=0$ or $\mu_{Y}=0$ (for speeding up), because points with 0 coordinate values can appear. In RLC, by choosing $\mu_{X}, \mu_{Y} \neq 0$, such special points will not appear in the process of scalar multiplications. This is the same against ZPA. Consequently, RLC resists SPA, DPA, RPA, and ZPA if the parameter set is chosen suitably (namely $\mu_{X}, \mu_{Y} \neq 0$ ). 
Table 8. ADA and RLC

\begin{tabular}{ll}
\hline INPUT: $d, P$. & OUTPUT: $d P$ \\
\hline $1:$ & $\mathrm{T}[0]=0, \mathrm{~T}[2]=\mathrm{RLC}(\mathrm{P})$ \\
$2:$ & for $i=0$ upto $\mathrm{n}-1\{$ \\
$3:$ & $\mathrm{T}[1]=\mathrm{LCECADD}(\mathrm{T}[0], \mathrm{T}[2])$ \\
$4:$ & $\mathrm{T}[2]=\mathrm{LCECDBL}(\mathrm{T}[2])$ \\
$5:$ & $\mathrm{T}[0]=\mathrm{T}[\mathrm{d}[\mathrm{i}]]$ \\
$6:$ & \\
$7:$ & return invRLC $(\mathrm{T}[0])$ \\
\hline
\end{tabular}

Table 9. ADA and RIP

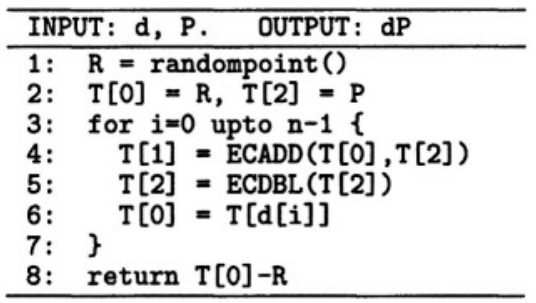

However, a straight-forward implementation of RLC may be vulnerable to RPA. For example, when we implement LCECADD in Table 7, direct computations of $X_{1}-\mu$ and $X_{2}-\mu$ should be avoided, because these values equal to 0 for a chosen base point $\bar{P}$ by RPA. Thus we have to compute $W, T$ without computing $\boldsymbol{X}_{\boldsymbol{i}}-\boldsymbol{\mu}$ and $\boldsymbol{U}_{\boldsymbol{i}}$. Realizations of concrete secure algorithms are shown in the following sections.

In the followings, we combine RLC and three addition chains concretely, and then discuss the security against possible attacks. As we will consider over a finite field with prime elements, the condition $\mu_{X} \neq 0$ should be satisfied.

Combination with Add-and-double-always Method from MSB. Applying RLC to the add-and-double-always method from MSB is easy (so we omit a concrete algorithm because of the space limitation). Here both LCECADD and LCECDBL should be implemented not to compute $\boldsymbol{X}_{\boldsymbol{i}}-\boldsymbol{\mu}_{\boldsymbol{X}}$ as mentioned before. Remember that if $\mu_{X}$ is chosen randomly (different from 0 ), the probability that $X_{i}-\mu_{X}=\mathbf{0}$ is negligible. Concrete secure algorithms LCECADD1 and LCECDBL1 for a parameter set $\boldsymbol{S}_{\mathbf{1}}=\left(\boldsymbol{\lambda}^{\mathbf{2}}, \lambda^{\mathbf{3}}, \lambda, \boldsymbol{\mu}, \mathbf{0}, \mathbf{0}\right), \boldsymbol{\mu} \in \boldsymbol{K}^{\mathbf{*}}$ are shown in Table Al and Table A2 in the appendix. Note that these algorithms are constructed not to output 0 values after each operation in order to resist ZPA. The processing speed of LCECADD1 is $14 M+4 S$ (Table Al) and LCECDBL1 is $8 M+7 S$ (Table A2). An average processing speed for each bit is $T^{\mathrm{MSB}}=(14 M+4 S)+(8 M+7 S)=22 M+11 S$.

Combination with Add-and-double-always Method from LSB. Applying RLC to the add-and-double-always method from LSB is also easy. A concrete algorithm is shown in Table 8. In this case, a variable $\mathrm{T}$ [2] has no relation to the secret key $\boldsymbol{d}$. Hence there is no need for $\mathrm{T}$ [2] to be protected. In other words, we are allowed to compute $\boldsymbol{X}_{\mathbf{2}}-\boldsymbol{\mu}_{\boldsymbol{X}}$ in LCECADD, and allowed not to use secure algorithm for LCECDBL. Thus we only take care not to compute $X_{1}-\mu_{X}$ in LCECADD. A concrete algorithm LCECADD1' for a parameter set $S_{1}=\left(\lambda^{2}, \lambda^{3}, \lambda, \mu, 0,0\right), \mu \in K^{*}$ is shown in Table A3 in the appendix. Note that the algorithm is constructed not to output 0 values after each operation in order to resist ZPA. The processing speed of LCECADD1' is $13 M+4 S$ (Table 
A1) and LCECDBL1 is $4 M+6 S$ (Table 3). An average processing speed for each bit is $T^{\mathrm{LSB}}=(13 M+4 S)+(4 M+6 S)=17 M+10 S$.

When the secret key $d$ has successive 0 in lowest bits, the number of 0 can be detected, because variables $\mathrm{T}[0], \mathrm{T}[2]$ are not randomized by insecure LCECDBL. Once 1 appears in $d, T[0]$ is randomized in LCECADD and no information will be leaked moreover. One solution for the leakage is to use secure LCECDBL and compute $X-\mu_{X}$ directly. Since the effect of $\lambda$ 's are remained, the security is improved without reducing the processing speed.

In the Jacobian coordinates, Itoh et al. proposed an efficient algorithm called the iterated ECDBL [13], which computes $2^{k} P$ for a given integer $k \geq 1$ more efficiently than computing ECDBL $k$ times by recycling intermediate values; the iterated ECDBL requires $4 k M+(4 k+2) S$, while each ECDBL requires $4 M+6 S$ (Table 4). In our countermeasure for the parameter set $S_{1}$, a variable T [2] only concerns a sequence $P, 2 P, 2^{2} P, \ldots$ and the iterated ECDBL can be applied efficiently. In this case, an averaged processing speed for each bit is optimized to $T_{0}^{\mathrm{LSB}}=(13 M+4 S)+(4 M+4 S)=17 M+8 S$.

Combination with Montgomery Ladder. Applying RLC to the Montgomery ladder is also easy. In this case, all variables T [0 ], T [1], T [2 ] depend on the secret key and both LCECADD and LCECDBL should be implemented not to compute $X_{i}-\mu_{X}$.

When we use secure algorithms LCECADD1, LCECDBL1 for the parameter set $S_{1}$ (Table A1 and Table A2 in the appendix), an average processing speed is $T^{\text {Mon }}=(14 M+4 S)+(6 M+6 S)=20 M+10 S$.

The Montgomery ladder provides good processing speed by using the specialized addition formula, the $x$-coordinate-only addition formula $[4,12,11]$, which computes ECADD and ECDBL with only $x$-coordinate value in the affine coordinates, or $X$ - and $Z$-coordinate values in the projective or the Jacobian coordinates. We can easily establish similar formulas XLCECADD, xLCECDBL, which compute ECADD and ECDBL in our linearly-transformed coordinates by the $x$-coordinate-only formula.

Moreover, Izu-Takagi proposed an encapsuled formula ECADDDBL, which takes $P_{1}, P_{2}$ on input and outputs $P_{1}+P_{2}$ and $2 P_{1}$, and XECADDDBL computed by the $x$-coordinate-only formula. In this case, an improved version of the Montgomery ladder is required [11]. A concrete algorithm XLCECADDDBL2, which computes XECADDDBL in LC by the $x$-coordinate-only method for a parameter set $S_{2}=(\lambda, \lambda, \lambda, \mu, 0,0)$, is shown in Table A4 (where the additive ECADD is used [12]). Here the algorithm is constructed not to output 0 values after each operation in order to resist ZPA, and the processing speed for each bit is $T_{o}^{\text {Mon }}=20 M+5 S$. 


\section{Randomized Initial Point Countermeasure (RIP)}

Next, we propose another countermeasure against power analysis.

Proposed Countermeasure. In the add-and-double always method from LSB (Table 2), an initial value of $\mathrm{T}$ [ 0 ] is set to $\mathcal{O}$. If this initial value is changed to a randomized point $R$, intermediate information will be randomized and thus it resists power analysis. (Of course, $R$ should be subtracted from a result of a scalar multiplication.) This countermeasure is called the randomized initial point countermeasure (RIP). A generic algorithm of RIP combined with Table 2 is shown in Table 9. Here a function randompoint () generates a random point on the curve. Note that RIP can be applied only to the add-and-double always method from LSB.

Selection of Initial Point. The security of RIP depends on how to select an initial point $R$. The simplest way is to generate the $x$-coordinate value of $R$ and compute corresponding $y$-coordinate value. One can obtain such $y$ with probability $1 / 2$, namely it might require large amount of time to obtain $R$. However, $R$ can be obtained in short time practically.

The other way is to convert a fixed and stored point $Q$ into a randomized point $R$ by using RPC. As the conversion is very cheap, it provides an efficient generation of $R$.

Security Consideration. Firstly, as RIP does not resist SPA (similarly to RLC), we have to use the add-and-double always method for the addition chain (Table 9). When an initial point $R$ is chosen properly, as a representation of $R$ would be randomized, RIP resists DPA (contrary, RIP is vulnerable to DPA if $R$ is fixed). Here there is no need to apply RPC or RC. On the other hand, RIP also resists RPA and ZPA, because the all intermediate values are randomized by the initial point $R$.

Processing Speed. When $R$ is set from $Q$ by RPC, the processing time of generating $R$ is negligible. Thus, the processing time of RIP per bit equals to a sum of that of ECADD and ECDBL. It is $(12 M+2 S)+(7 M+5 S)=19 M+$ $7 S$ for the projective coordinates, and $(12 M+4 S)+(4 M+6 S)=16 M+10 S$ for the Jacobian coordinates.

When the Jacobian coordinates are used, the iterated ECDBL can be also applied similarly to RLC. In this case, the processing time of RIP per a bit equals $(12 M+4 S)+(4 M+6 S)-2 S=16 M+8 S$. 
Table 10. Comparison of secure countermeasures

\begin{tabular}{l|c|rc}
\hline \multicolumn{1}{c|}{ Method } & Coordinates & \multicolumn{2}{|c}{ Speed in each bit } \\
\hline iES [7] & $\mathcal{J}$ & $16 M+10 S$ & $(24.0 M)$ \\
iES with Montgomery's trick & $\mathcal{J}$ & $12 M+9 S$ & $(19.2 M)$ \\
\hline RLC with MSB & $\mathcal{J}$ & $22 M+11 S$ & $(30.2 M)$ \\
RLC with LSB & $\mathcal{J}$ & $17 M+10 S$ & $(25.0 M)$ \\
RLC with LSB and iECDBL & $\mathcal{J}$ & $17 M+8 S$ & $(23.4 M)$ \\
RLC with Montgomery ladder & $\mathcal{J}$ & $20 M+5 S$ & $(24.0 M)$ \\
\hline RIP with LSB & $\mathcal{P}$ & $19 M+7 S$ & $(24.6 M)$ \\
RIP with LSB & $\mathcal{J}$ & $16 M+10 S$ & $(24.0 M)$ \\
RIP with LSB and iECDBL & $\mathcal{J}$ & $16 M+8 S$ & $(22.4 M)$ \\
\hline
\end{tabular}

\section{Comparison}

Let us compare countermeasures against power analysis that resist all of SPA, DPA, RPA and ZPA. We provide practical processing time for the improved exponent splitting (iES) [7] and our proposed two countermeasures (RLC and RIP). Processing speed are given averaged for each bit of the scalar $d$ and is estimated with the assumption that $1 S=0.8 M[27,12]$.

As in the table, iES with Shamir's trick and Montgomery's trick provides the most efficient processing speed. However, our proposed countermeasures provide comparable speed. A difference between iES and RIP is the number of registers for points on the curve; $\mathrm{iES}$ requires 5 registers while our RIP requires only 4 registers for a scalar multiplication. Note that, in RLC, the seed of an initial point $R$ is required. That is, RLC requires extra memory compared to other methods. On the other hand, RLC requires no extra memory.

\section{Concluding Remarks}

In this paper, two new practical countermeasures against power analysis are proposed, the randomized linearly-transformed coordinates countermeasure and the randomized initial point countermeasure. These methods improves the processing speed without reducing the security.

Since RPA and ZPA are newer attacks, the property of the attacks and of the countermeasures are not well studied yet. Theoretically, iES provides a better processing speed, however, because it uses a small table of pre-computed points, the resistance against the address-bit DPA [10] and the 2nd order DPA [28] should be analyzed. On the other hand, applying RIP to other addition chains will be for future work. Very recently, Morimoto-Mamiya-Miyaji attempted to apply RIP to the binary method from MSB [23], for which further analysis will be required. 


\section{Acknowledgments}

The authors would like to thank Toru Akishita, JJean-Bernard Fischer and Naoya Torii for their valuable and helpful comments on the preliminary version of this paper. We also thank anonymous reviewers of the conference for introducing required references.

\section{References}

[1] R. Avanzi, "Countermeasures against Differential Power Analysis for Hyperelliptic Curve Cryptosystems", CHES 2003, LNCS 2779, pp.366-381, Springer-Verlag, 2003.

[2] T. Akishita, and T. Takagi, "Zero-value Point Attacks on Elliptic Curve Cryptosystem", ISC 2003, LNCS 2851, pp.218-233, Springer-Verlag, 2003.

[3] T. Akishita, and T. Takagi, "On the Optimal Parameter Choice for Elliptic Curve Cryptosystems Using Isogeny”, PKC 2004, LNCS 2947, pp.346-359, Springer-Verlag, 2004.

[4] E. Brier, and M. Joye, "Weierstraß Elliptic Curves and Side-Channel Attacks", $P K C$ 2002, LNCS 2274, pp.335-345, Springer-Verlag, 2002.

[5] J. Coron, "Resistance against Differential Power Analysis for Elliptic Curve Cryptosystem", CHES'99, LNCS 1717, pp.292-302, Springer-Verlag, 1999.

[6] C. Clavier, and M. Joye, "Universal exponentiation algorithm - A first step towards provable SPA-resistance-”, CHES 2001, LNCS 2162, pp. 300-308, Springer-Verlag, 2001.

[7] M. Ciet, and M. Joye, "(Virtually) Free Randomization Technique for Elliptic Curve Cryptography”, ICICS 2003, LNCS 2836, pp. 348-359, Springer-Verlag, 2003.

[8] H. Cohen, A. Miyaji, and T. Ono, "Efficient Elliptic Curve Exponentiation Using Mixed Coordinates”, Asiacrypt'98, LNCS 1514, pp.51-65, Springer-Verlag, 1998.

[9] L. Goubin, "A Refined Power-Analysis Attack on Elliptic Curve Cryptosystems", $P K C$ 2003, LNCS 2567, pp.199-210, Springer-Verlag, 2003.

[10] K. Itoh, T. Izu, M. Takenaka, "Address-bit Differential Power Analysis of Cryptographic Schemes OK-ECDH and OK-ECDSA", CHES 2002, LNCS 2523, pp.129-143,2003.

[11] T. Izu, B. Möller, and T. Takagi, "Improved Elliptic Curve Multiplication Methods Resistant against Side Channel Attacks", Indocrypt 2002, LNCS 2551, pp.296-313, SpringerVerlag, 2002.

[12] T. Izu, and T. Takagi, "A Fast Parallel Elliptic Curve Multiplication Resistant against Side Channel Attacks”, PKC 2002, LNCS 2274, pp.280-296, Springer-Verlag, 2002.

[13] K.Itoh, M.Takenaka, N.Torii, S.Temma, and Y.Kurihara, "Fast Implementation ofPublicKey Cryptography on DSP TMS320C6201”, CHES'99, LNCS 1717, pp.61-72,1999.

[14] K. Itoh, J. Yajima, M. Takenaka, and N. Torii, "DPA Countermeasures by Improving the Window Method", CHES 2002, LNCS 2523, pp.303-317, Springer-Verlag, 2003.

[15] M. Joye, C. Tymen, "Protections against Differential Analysis for Elliptic Curve Cryptography", CHES 2001, LNCS 2162, pp.377-390, Springer-Verlag, 2001.

[16] M. Joye, and S-M. Yen, "The Montgomery Powering Ladder", CHES 2002, LNCS 2523, pp.291-302, Springer-Verlag, 2003.

[17] C. Kocher, "Timing Attacks on Implementations of Diffie-Hellman, RSA, DSS, and Other Systems", Crypto'96, LNCS 1109,pp.104-113, Springer-Verlag, 1996. 
[18] C. Kocher, J. Jaffe, and B. Jun, "Differential Power Analysis”, Crypto'99, LNCS 1666, pp.388-397, Springer-Verlag, 1999.

[19] B. Möller, "Securing Elliptic Curve Point Multiplication against Side-Channel Attacks", ISC 2001, LNCS 2200, pp.324-334, Springer-Verlag, 2001.

[20] B. Möller, "Parallelizable Elliptic Curve Point Multiplication Method with Resistance against Side-Channel Attacks", ISC 2002, LNCS 2433, pp.402-413, Springer-Verlag, 2002.

[21] P. Montgomery, "Speeding the Pollard and Elliptic Curve Methods for Factorizations", Math. of Comp, vol.48, pp.243-264, 1987.

[22] T. Messerges, E. Dabbish, and R. Sloan, "Power Analysis Attacks of Modular Exponentiation in Smartcards", CHES'99, LNCS 1717, pp. 144-157, Springer-Verlag, 1999.

[23] H. Morimoto, H. Mamiya, and A. Miyaji, "Elliptic Curve Cryptosystems Secure against ZPA"(in Japanese), Technical Report of the Institute of Electronicas, Information and Communication Engineers (IEICE), ISEC 2003-103, March, 2004. English version is to appear in the proceedings of CHES 2004.

[24] Recommended Elliptic Curves for Federal Government Use, in the appendix of FIPS 186-2, National Institute of Standards and Technology (NIST).

[25] E. Oswald, and M. Aigner, "Randomized Addition-Subtraction Chains as a Countermeasure against Power Attacks", CHES 2001, LNCS 2162, pp.39-50, Springer-Verlag, 2001.

[26] K. Okeya, H. Kurumatani, and K. Sakurai, "Elliptic curves with the Montgomery form and their cryptographic applications", PKC 2000, LNCS 1751, pp.446-465, SpringerVerlag, 2000.

[27] K. Okeya, and K. Sakurai, "Power analysis breaks elliptic curve cryptosystem even secure against the timing attack", Indocrypt 2000, LNCS 1977, pp. 178-190, Springer-Verlag, 2000.

[28] K. Okeya, and K. Sakurai, "On Insecurity of the Side Channel Attack Countermeasure Using Addition-Subtraction Chains under Distinguishability between Addition and Doubling”, ACISP 2002, LNCS 2384, pp.420-435, Springer-Verlag, 2002.

[29] K. Okeya, and K. Sakurai, "A Multiple Power Analysis Breaks the Advanced Version of the Randomized Addition-Subtraction Chains Countermeasure against Side Channel Attacks", to appear in the proceedings of 2003 IEEE Information Theory Workshop.

[30] K. Okeya, and T. Takagi, "The Width-w NAF Method Provides Small Memory", CT-RSA 2003, LNCS 2612, pp.328-342, Springer-Verlag, 2003.

[31] K. Okeya, and T. Takagi, "A More Flexible Countermeasure against Side Channel Attacks using Window Method”, CHES 2003, LNCS 2779, pp. 397-410 Springer-Verlag, 2003.

[32] N. Smart, “An Analysis of Goubin's Refined Power Analysis Attack”, CHES 2003, LNCS 2779, pp.281-290, Springer-Verlag, 2003.

[33] C. Walter, "Security Constraints on the Oswald-Aigner Exponentiation Algorithm", Cryptology ePrint Archive, Report 2003/013, 2003.

\section{Appendix: Algorithms for Proposed Countermeasures}

The appendix shows detailed concrete algorithms for LCECADD1, LCECDBL1, LCECADD1', and XLCECADDDBL2 for RLC introduced in section 4.1. The parameter set for LCECADD1, 
Table Al. ECADD in LC with a parameter set $S_{1}=\left(\lambda^{2}, \lambda^{3}, \lambda, \mu, 0,0\right)$

Table A2. ECDBL in LC with a parameter set LCECADD1 (in 14M+4S)

\section{Input: $P_{1}=\left(X_{1}, Y_{1}, Z_{1}\right)$, \\ $P_{2}=\left(X_{2}, Y_{2}, Z_{2}\right), \mu$}

Output: $P_{3}=P_{1}+P_{2}=\left(X_{3}, Y_{3}, Z_{3}\right)$

\begin{tabular}{|c|c|}
\hline $\begin{array}{l}T_{1} \leftarrow Z_{1}^{2} \\
T_{2} \leftarrow Z_{1} \times T_{1} \\
T_{3} \leftarrow Z_{2}^{2} \\
T_{4} \leftarrow Z_{2} \times T_{3} \\
T_{5} \leftarrow X_{1} \times T_{3} \\
T_{6} \leftarrow \mu \times T_{3} \\
T_{7} \leftarrow X_{2} \times T_{1} \\
T_{8} \leftarrow \mu \times T_{1} \\
T_{9} \leftarrow Y_{1} \times T_{4} \\
T_{10} \leftarrow Y_{2} \times T_{2} \\
T_{11} \leftarrow T_{7}-T_{5} \\
T_{12} \leftarrow T_{11}-T_{8} \\
T_{13} \leftarrow T_{12}+T_{6} \\
T_{14} \leftarrow T_{7}+T_{5} \\
T_{15} \leftarrow T_{14}-T_{8} \\
T_{16} \leftarrow T_{15}-T_{6} \\
T_{17} \leftarrow T_{10}-T_{9} \\
T_{18} \leftarrow T_{10}+T_{9} \\
T_{19} \leftarrow T_{17}^{2} \\
T_{20} \leftarrow T_{13}^{2} \\
T_{21} \leftarrow T_{16} \times T_{20} \\
T_{22} \leftarrow T_{19}+\mu \\
X_{3} \leftarrow T_{22}-T_{21} \\
T_{23} \leftarrow 2 * X_{3} \\
T_{24} \leftarrow T_{23}-T_{21} \\
T_{25} \leftarrow 2 * \mu \\
T_{26} \leftarrow T_{25}-T_{24} \\
T_{27} \leftarrow T_{25} \times T_{17} \\
T_{28} \leftarrow T_{18} \times T_{13} \\
T_{29} \leftarrow T_{28} \times T_{20} \\
2 Y_{3} \leftarrow T_{27}-T_{29} \\
T_{30} \leftarrow Z_{1} \times Z_{2} \\
Z_{3} \leftarrow T_{30} \times T_{13}\end{array}$ & $\begin{array}{l}\left(=Z_{1}^{2}\right) \\
\left(=Z_{1}^{3}\right) \\
\left(=Z_{2}^{2}\right) \\
\left(=Z_{2}^{3}\right) \\
\left(=X_{1} Z_{2}^{2}\right) \\
\left(=\mu Z_{2}^{2}\right) \\
\left(=X_{2} Z_{1}^{2}\right) \\
\left(=\mu Z_{1}^{2}\right) \\
\left(=Y_{1} Z_{2}^{3}=S_{1}\right) \\
\left(=Y_{2} Z_{1}^{3}=S_{2}\right) \\
\left(=X_{2} Z_{1}^{2}-X_{1} Z_{2}^{2}\right) \\
\left(=U_{2}-X_{1} Z_{2}^{2}\right) \\
\left(=U_{2}-U_{1}=W\right) \\
\left(=X_{2} Z_{1}^{2}+X_{1} Z_{2}^{2}\right) \\
\left(=U_{2}+X_{1} Z_{2}^{2}\right) \\
\left(=U_{2}+U_{1}=T\right) \\
\left(=S_{2}-S_{1}=R\right) \\
\left(=S_{2}+S_{1}=M\right) \\
\left(=R^{2}\right) \\
\left(=W^{2}\right) \\
\left(=T W^{2}\right) \\
\left(=R^{2}+\mu\right) \\
\left(=R^{2}-T W^{2}+\mu\right)\end{array}$ \\
\hline
\end{tabular}

$S_{1}=\left(\lambda^{2}, \lambda^{3}, \lambda, \mu, 0,0\right)$

LCECDBL1 (in 8M+7S)

Input: $P_{1}=\left(X_{1}, Y_{1}, Z_{1}\right), a, \mu$

Output: $P_{4}=2 P_{1}=\left(X_{4}, Y_{4}, Z_{4}\right)$

\begin{tabular}{|c|c|}
\hline $\begin{array}{l}T_{1} \leftarrow Z_{1}^{2} \\
T_{2} \leftarrow T_{1}^{2} \\
T_{3} \leftarrow a \times T_{2} \\
T_{4} \leftarrow X_{1}^{2} \\
T_{5} \leftarrow 2 * X_{1} \\
T_{6} \leftarrow \mu-T_{5} \\
T_{7} \leftarrow \mu \times T_{6} \\
T_{8} \leftarrow 3 * T_{4} \\
T_{9} \leftarrow 3 * T_{7} \\
T_{10} \leftarrow T_{3}+T_{8} \\
T_{11} \leftarrow Y_{1}^{2} \\
T_{12} \leftarrow X_{1} \times T_{1} \\
T_{13} \leftarrow \mu \times T_{11} \\
T_{14} \leftarrow T_{11}^{2} \\
T_{15} \leftarrow 8 * T_{14} \\
T_{16} \leftarrow T_{9}^{2} \\
T_{17} \leftarrow T_{9} \times T_{10} \\
T_{18} \leftarrow 2 * T_{17} \\
T_{19} \leftarrow T_{10}^{2} \\
T_{20} \leftarrow \mu+T_{16} \\
T_{21} \leftarrow T_{20}+T_{1} \\
T_{22} \leftarrow T_{21}+T_{1} \\
T_{23} \leftarrow 8 * T_{12} \\
T_{24} \leftarrow 8 * T_{13} \\
T_{25} \leftarrow T_{22}-T_{2} \\
X_{4} \leftarrow T_{22}+T_{24} \\
T_{26} \leftarrow 4 * T_{12} \\
T_{27} \leftarrow 4 * T_{13} \\
T_{28} \leftarrow T_{26}+\mu \\
T_{29} \leftarrow X_{4}-T_{28} \\
T_{30} \leftarrow T_{29}-T_{2} \\
T_{31} \leftarrow T_{9} \times T_{30} \\
T_{32} \leftarrow T_{10} \times T_{3} \\
T_{33} \leftarrow T_{31}-T_{1} \\
Y_{4} \leftarrow T_{33}+T_{32} \\
T_{34} \leftarrow Y_{1} \times Z_{1} \\
Z_{4} \leftarrow 2 * T_{34} \\
\end{array}$ & $\begin{array}{l}\left(=\left(W+3 X_{1}^{2}\right)^{2}\right) \\
8 \\
9\left(=\mu+M^{2}\right) \\
\left(=8 X_{1} Y_{1}^{2}\right) \\
\left(=8 \mu Y_{1}^{2}\right) \\
3\left(=M^{2}-8 X_{1} Y_{1}^{2}+\mu\right) \\
\left(=M^{2}-2 S+\mu\right) \\
\left(=4 X_{1} Y_{1}^{2}\right) \\
\left(=4 \mu Y_{1}^{2}\right) \\
\left(=4 X_{1} Y_{1}^{2}+\mu\right) \\
\left(=4 X_{1} Y_{1}^{2}-X_{4}+\mu\right) \\
\left.7=S-X_{4}+\mu\right) \\
\left(={ }^{2}\right) \\
5 \\
\left(=M\left(S-X_{4}+\mu\right)-T\right) \\
\left(=Y_{1} Z_{1}\right) \\
\left(=2 Y_{1} Z_{1}\right)\end{array}$ \\
\hline
\end{tabular}

LCECDBL1, LCECADD1' is $\boldsymbol{S}_{\mathbf{1}}=\left(\boldsymbol{\lambda}^{\mathbf{2}}, \boldsymbol{\lambda}^{\mathbf{3}}, \boldsymbol{\lambda}, \boldsymbol{\mu}, \mathbf{0 , 0}\right)$ and that for xLCECADDDBL2 is $\boldsymbol{S}_{\mathbf{2}}=$ $(\lambda, \lambda, \lambda, \mu, 0,0)$. In the algorithms, $\times$ denotes a general multiplication, while $*$ denotes a multiplication by a constant. We ignore the processing time for $*$ in the evaluations. 